\title{
What Does Low Energy Physics Tell Us About The Zero Momentum Gluon Propagator
}

\author{
P. Costa ${ }^{1}$, O. Oliveira ${ }^{1}$, and P. J. Silva ${ }^{1}$ \\ 1 Departamento de Física, Universidade de Coimbra, P-3004-516 Coimbra, Portugal
}

\begin{abstract}
The connection between QCD, a nonlocal Nambu-Jona-Lasinio type model and the Landau gauge gluon propagator is explored. This two point function is parameterized by a functional form which is compatible with Dyson-Schwinger and lattice QCD results. Demanding the nonlocal model to reproduce the experimental values for the pion mass, the pion decay constant, $\Gamma_{\pi \rightarrow \gamma \gamma}$ and the light quark condensate we conclude that low energy physics does not distinguish between the so-called decoupling and scaling solutions of the Dyson-Schwinger equations. This result means that, provided that the model parameters are chosen appropriately, one is free to chose any of the above scenarios. Furthermore, the nonlocal Nambu-Jona-Lasinio quark model considered here is chiral invariant and satisfies the GMOR relation at the $1 \%$ level of precision.
\end{abstract}

Keywords: Gluon propagator, chiral symmetry, lattice QCD 
The usual approach to Quantum Chromodynamics (QCD) defines the Green's function generation function à la Faddeev-Popov (FP) [1]. This approach allows the investigation of the perturbative solution of QCD. However, in what concerns nonperturbative phenomena, like confinement and chiral symmetry breaking, the FP trick is ill defined as it assumes a unique solution of the gauge fixing condition on each gauge orbit. Gribov demonstrated [2], for the Coulomb and Landau gauges, that in each set of gauge related configurations there are more than one configuration, the Gribov copies, satisfying the gauge fixing condition. The study of Gribov was confirmed by many lattice simulations [3], which also investigate how the copies modify the Green's functions. Furthermore, there is a no-go theorem [4] stating that the problem of the copies is common to all local gauge fixing conditions.

Gribov tried to solve the problem of the copies by changing the functional integration space, replacing the hyperplane of transverse configurations, $\Gamma=\{A: \partial \cdot A=0\}$, by the first Gribov region $\Omega$ : the subset of $\Gamma$ with a positive defined Faddeev-Popov operator. This change of integration space modifies the tree level gluon propagator in the infrared region and, according to Gribov, at zero momentum the propagator vanishes. However, the problem of building a nonperturbative generating functional for QCD was not solved, as $\Omega$ is not free of Gribov copies.

In [5, 6] the restriction of the integration space to $\Omega$ was implemented via a modification of the QCD action. The Gribov-Zwanziger (GZ) action includes extra bosonic and fermionic fields, breaks softly BRST [6, 7] and is perturbatively renormalizable. Moreover, the GZ action also predicts a vanishing zero momentum gluon propagator [8]. This result suggested a gluonic confinement criteria as discussed in [5, 9]. Further extensions of the GZ action, including possible condensates, were investigated in [7] leading to the so-called refined Gribov-Zwanziger (RGZ) action. If the GZ action predicts a $D(0)=0$, according to the RGZ action $D(0) \neq 0\lfloor 10]$.

In the recent years there has been a considerable effort to compute the gluon and ghost propagators from first principles, namely using Dyson-Schwinger equations (DSE) and the lattice formulation of QCD. Considerable attention was given to the infrared propagators and to the value of $D(0)$.

DSE computations start from the Faddeev-Popov quantization procedure. The solutions follow into two categories: the scaling solution [11, 12] which has $D(0)=0$ and is compatible with the Gribov-Zwanziger confinement criterium; the decoupling solution [13-15] which 
predicts a $D(0) \neq 0$, related to a dynamical generated gluon mass $M\left(p^{2}\right)$.

Wilson action 4D lattice QCD simulations for the gluon and ghost propagators have been performed for $\mathrm{SU}(2)$ and $\mathrm{SU}(3)$ gauge groups [16 23]. The gluon propagator has been computed for volumes as large as $(27 \mathrm{fm})^{4}$ for $\mathrm{SU}(2)[21](a \sim 0.21 \mathrm{fm})$, and $(16 \mathrm{fm})^{4}$ for $\mathrm{SU}(3)$ [22] ( $a \sim 0.17 \mathrm{fm})$. The raw data shows a decreasing $D(0)$ with the lattice volume but no infrared suppression of $D\left(p^{2}\right)$ has been observed. Furthermore, naive extrapolations of $D(0)$ to the infinite volume [20] do not provide a clear answer about the infinite volume value for $D(0)$.

In [21], inequalities relating the zero momentum gluon propagator with an average absolute value color-magnetization, which allow for a scaling analysis with the volume, were derived. $\mathrm{SU}(2)$ simulations [21] give a finite and nonvanishing $D(0)$ in the limit $V \rightarrow+\infty$. A similar analysis for $\mathrm{SU}(3)$ [23], although using smaller volumes, give $D(0)=0$ in the same limit. The reasons for this difference are still not understood.

In [24] the authors considered ratios of lattice propagators, which, hopefully, suppress the finite volume effects. The analysis of the ratios point towards a vanishing $D(0)$ in the infinite volume limit.

In conclusion, the present situation gives ambiguous results for the value of $D(0)$. This question is of great theoretical relevance as it validates, or not, possible gluon confinement mechanisms. Any other way of estimating $D(0)$ independently is welcome. In this work we link $D(0)$ with low energy physics, i.e. the physics associated with pions. In order to connect the infrared gluon propagator with low energy phenomenology, an effective low energy chiral quark model of the Nambu-Jona-Lasinio type is built.

In the QCD Lagrangian, the interaction between quarks and gluons reads

$$
\mathcal{L}_{\bar{\psi} \psi A}=g \bar{\psi} \gamma^{\mu} A_{\mu}^{a} \frac{\lambda^{a}}{2} \psi
$$

In the generating functional, expanding the exponential term containing $\mathcal{L}_{\bar{\psi} \psi A}$ to second order in $g$ and integrating the gluon fields, the theory becomes an effective nonlocal fermionic theory. For one flavor QCD, after color and spin Fierz transformations, assuming that vector and axial vector currents play a secondary role in the dynamics, and taking into account only the color singlet bilocal currents $J(x, y)=\bar{\psi}^{i}(x) \psi^{i}(y), J_{5}(x, y)=\bar{\psi}^{i}(x) \gamma_{5} \psi^{i}(y)(i$ stands 


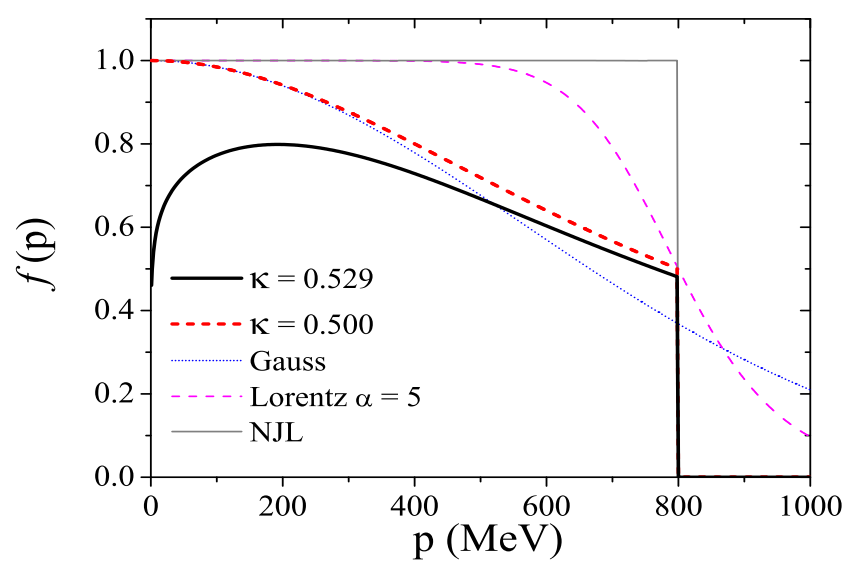

FIG. 1. Form factors as a function of $p$. The figure includes typical form factors used in previous studies - see, for example, [26] and references there in.

for color indices) one gets the effective action

$$
\begin{aligned}
S[\bar{\psi}, \psi] & =\int d^{4} x d^{4} y\left\{\bar{\psi}(y) \delta(y-x)\left(i \gamma^{\mu} \partial_{\mu}-m\right) \psi(x)\right. \\
& \left.+\frac{g^{2}}{8} J(x, y) D(x-y) J(y, x)-\frac{g^{2}}{8} J_{5}(x, y) D(x-y) J_{5}(y, x)\right\} .
\end{aligned}
$$

This action is invariant under global chiral symmetry, i.e. $\psi \longrightarrow e^{i \theta \gamma_{5}} \psi$ and $\bar{\psi} \longrightarrow \bar{\psi} e^{-i \theta \gamma_{5}}$ , provided that $m=0$, and is sensible to the details of the gluon propagator $D(x-y)$. Extending (2) to include various quark flavors is straightforward. In the following it will be assumed $\mathrm{SU}(2)$-flavor symmetry and the current quark masses will be written as $m_{u}=$ $m_{d}=m_{q}$.

The action (22) is a nonlocal version of the Nambu-Jona-Lasinio (NJL) action [25]. The NJL model gives a good description of low energy hadron phenomenology and it is expected that the nonlocal version should do a similar job, with the extra bonus of being sensible to the gluon propagator. The NJL model is nonrenormalizable and needs to be regularized by the introduction of a finite cut-off $\Lambda$. For the local version, where $D(x-y) \propto \delta(x-y)$, typical cut-off values range from $600 \mathrm{MeV}$ up to $800 \mathrm{MeV}$. For the model described by Eq. (21), besides the regularization, one also needs to know the gluon form factor $D(x-y)$.

First principles calculations of the gluon propagator have been performed using lattice 
QCD and DSE. The momentum space propagator

$$
D\left(p^{2}\right)=Z \frac{\left(p^{2}\right)^{2 \kappa-1}}{\left(p^{2}+\Lambda_{Q C D}^{2}\right)^{2 \kappa}}
$$

is able to describe both the scaling $(\kappa>0.5)$ and decoupling $(\kappa=0.5)$ infrared DSE solutions and the lattice data up to $p \sim 800 \mathrm{MeV}$ - see Refs. [17, 18] for discussions. In Eq. (3), $\Lambda_{Q C D}$ stands for an infrared mass scale.

Let us define the dimensionless form factor in momentum space as

$$
f\left(p^{2}\right)=\Lambda^{2} D\left(p^{2}\right)=\frac{\Lambda^{2}}{p^{2}}\left(\frac{p^{2}}{p^{2}+\Lambda_{Q C D}^{2}}\right)^{2 \kappa} \theta(\Lambda-p) .
$$

The constant $Z$ in Eq. (3) will be included in the definition of the coupling constant $G$, which multiplies the quark currents of the nonlocal theory. $G$ carries the dimension of a length squared. In $f\left(p^{2}\right), \Lambda$ is the cut-off. In a first step we assume $\Lambda_{Q C D}=\Lambda$. The form factor $f\left(p^{2}\right)$, together with typical form factors considered in the literature, is shown in Fig. 1

The model has four parameters $\left\{G, m_{q}, \kappa, \Lambda\right\}$ to be determined in vacuum. Given that Eq. (3) reproduces the lattice data for $p<800 \mathrm{MeV}$, we take $\Lambda=800 \mathrm{MeV}$. For each $\kappa, G$ and $m_{q}$ are adjusted to reproduce the experimental pion mass $M_{\pi}=139.57 \mathrm{MeV}$ and decay constant $f_{\pi}=92.4 \mathrm{MeV}$.

We also take in consideration the light quark condensate $\langle\bar{q} q\rangle$. At a renormalization scale of $\mu=1 \mathrm{GeV},\langle\bar{q} q\rangle^{1 / 3}=-240 \mathrm{MeV}$, while at $\mu=2 \mathrm{GeV}$ it becomes $\langle\bar{q} q\rangle^{1 / 3}=-270 \mathrm{MeV}$ [27, 28]. The difference is mainly due to the running of $\hat{m}$ from $5.5 \mathrm{MeV}$, for $\mu=1 \mathrm{GeV}$, to $4.1 \mathrm{MeV}$ if $\mu=2 \mathrm{GeV}$ - see Ref. [29]. Furthermore, due to phenomenological reasons, we will restrict our study to constituent quark masses $M_{q}$ in the range $300-400 \mathrm{MeV}$, which are typical values of $M_{q}$ in this type of models. The decay width for $\pi \longrightarrow \gamma \gamma$ will be used to distinguish the different sets of parameters.

Our nonlocal model belongs to a class of models which have been explored by previous authors [30 32] and we will not discuss how to compute the different quantities. The technical details can be found in [30].

We start the discussion of the results with a technical comment on the NJL-type model calculations. The fermionic action of NJL-type models has ultraviolet divergences and requires a regularizing cut-off [33]. However, not all the integrals are divergent and some 


\begin{tabular}{|c|c|c|c|c|c|}
\hline \hline & $m_{q}[\mathrm{MeV}]$ & $M_{q}[\mathrm{MeV}]$ & $-\langle\bar{q} q\rangle^{1 / 3}[\mathrm{MeV}]$ & $G \Lambda^{2}$ & $\Gamma_{\pi \gamma \gamma}[\mathrm{eV}]$ \\
\hline \hline$\kappa=0.40$ & 4.093 & 295.8 & 273.3 & 3.781 & 2.06 \\
\hline$\kappa=0.45$ & 4.146 & 325.6 & 272.2 & 4.946 & 3.22 \\
\hline$\kappa=0.50$ & 4.187 & 360.5 & 271.4 & 6.441 & 5.44 \\
\hline$\kappa=0.529$ & 4.205 & 383.6 & 271.1 & 7.491 & 7.79 \\
\hline$\kappa=0.548$ & 4.214 & 400.1 & 271.0 & 8.263 & 10.19 \\
\hline$\kappa=0.60$ & 4.231 & 453.3 & 271.4 & 10.776 & 26.20 \\
\hline
\end{tabular}

TABLE I. A collection of parameters which reproduce the experimental $M_{\pi}$ and $f_{\pi}$ for a cut-off $\Lambda=\Lambda_{Q C D}=800 \mathrm{MeV}$. The results differ essentially on the value of the decay width $\Gamma_{\pi \rightarrow \gamma \gamma}$ (last column). Recall that the experimental width is $7.78(56) \mathrm{eV}$.

authors chose to regularize only the action and the divergent integrals: for the finite integrals they remove the cut-off. This has an important impact on $\Gamma_{\pi \rightarrow \gamma \gamma}$. Indeed, for the local NJL model the width computed with all integrals regularized give, typically, only about $70 \%$ of the experimental numbers (see, for example, [31, 34] ). In our calculation the cut-off $\Lambda=800$ $\mathrm{MeV}$ was used at all the stages of the computation.

We would like to remember the reader that our work has two main goals. On one side we would like to define a nonlocal model for low energy physics which is, as much as possible, rooted in first principle computations. This motivates our choice for the form factor. On the other side, we would like to connect low energy physics with the behavior of the gluon propagator $D\left(p^{2}\right)$ in the infrared region and, in particular, its value for $p=0$.

In what concerns $M_{\pi}$ and $f_{\pi}$, for a cut-off of $\Lambda=800 \mathrm{MeV}$, it turns out that the experimental values can be reproduced by a large set of parameters, including $\kappa$ above and below 0.5 - see Table \. The values on Table \ show that the quark condensate is almost independent of $\kappa$ and always close to the expected value $\langle\bar{q} q\rangle^{1 / 3}=-270 \mathrm{MeV}$. It is important to point out that this model is consistent with the Gell-Mann-Oakes-Renner relation (GMOR)

$$
M_{\pi}^{2} f_{\pi}^{2}=-2 m_{q}\langle\bar{q} q\rangle_{0}
$$

preserving chiral low-energy theorems and current algebra relations [26]. As matter of fact, 


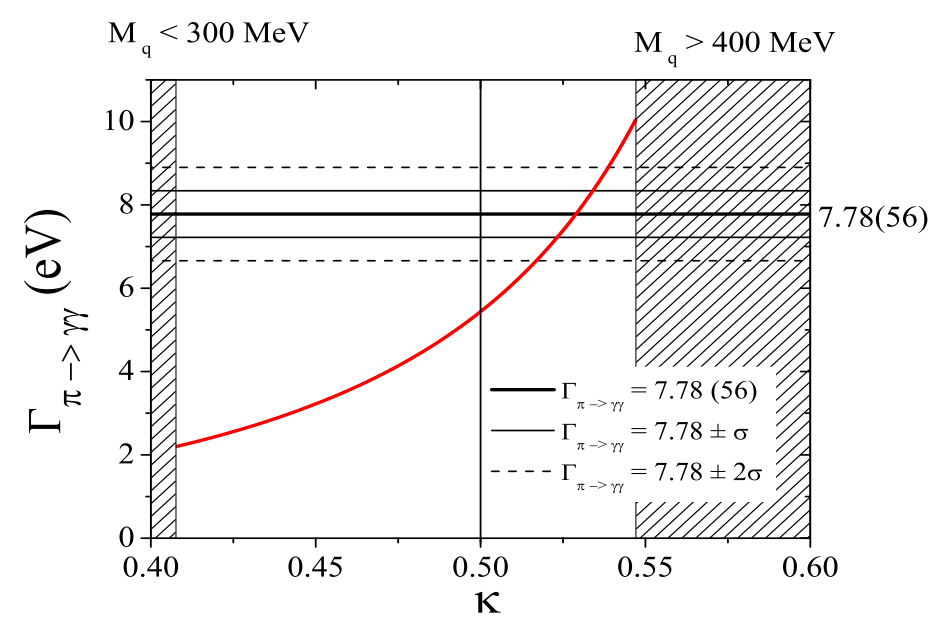

FIG. 2. $\Gamma_{\pi \rightarrow \gamma \gamma}$ as a function of $\kappa$ for $\Lambda=\Lambda_{Q C D}=800 \mathrm{MeV}$. The excluded regions, $\kappa<0.41$ and $\kappa>0.55$, are associated with a constituent quark mass outside typical phenomenological values $M_{q}<300 \mathrm{MeV}$ or $M_{q}>400 \mathrm{MeV}$. See also Table @

we can calculate the GMOR value for the current quark mass

$$
m_{q}^{G M O R}=-\frac{M_{\pi}^{2} f_{\pi}^{2}}{2\langle\bar{q} q\rangle_{0}},
$$

as an indicator of the validity of this low energy theorem. Taking for example $\kappa=0.5$, $m_{q}^{\text {GMOR }}=4.159 \mathrm{MeV}$ which differs less then $1 \%$ of the calculated value $m_{q}=4.187 \mathrm{MeV}$.

The width $\Gamma_{\pi \rightarrow \gamma \gamma}$, with an experimental value of 7.78 (56) eV [35], distinguishes between the various scenarios for $D(0)$ - see Fig. 2. Clearly, the two pion decay points towards a $\kappa$ above 0.5 and a $D(0)=0$. This conclusion is unchanged when $\Lambda \neq \Lambda_{Q C D}$ and for other cut-off values - see Fig. 3 .

The results reported in Fig. 3 show that to reproduce the experimental $\Gamma_{\pi \rightarrow \gamma \gamma}$, the cut-off $\Lambda$ and the infrared mass scale $\Lambda_{Q C D}$ are correlated. The cut-off increases with $\Lambda_{Q C D}$, with $\Lambda$ and $\Lambda_{Q C D}$ being of the same order of magnitude. Moreover, for each $\Lambda_{Q C D}$, there is only a narrow band of cut-off values which are able to reproduce the experimental width. For example, for $\Lambda_{Q C D}=750 \mathrm{MeV}, \Lambda$ has to be in the interval 761-885 MeV to reproduce $\Gamma_{\pi \rightarrow \gamma \gamma}$ up to two standard deviations. Furthermore, $\kappa$ seems to decrease slightly when $\Lambda_{Q C D}$ increases. Given the correlation between $\Lambda$ and $\Lambda_{Q C D}$, this suggests that for higher values of the cut-off, in principle, one could reproduce the full set of observables for $\kappa=0.5$ or smaller. However, since our nonlocal model does not take into account the vector and axial-vector bilocal quark currents, that the light $J=1$ spectrum starts at $\sim 800 \mathrm{MeV}$, an upper limit of 

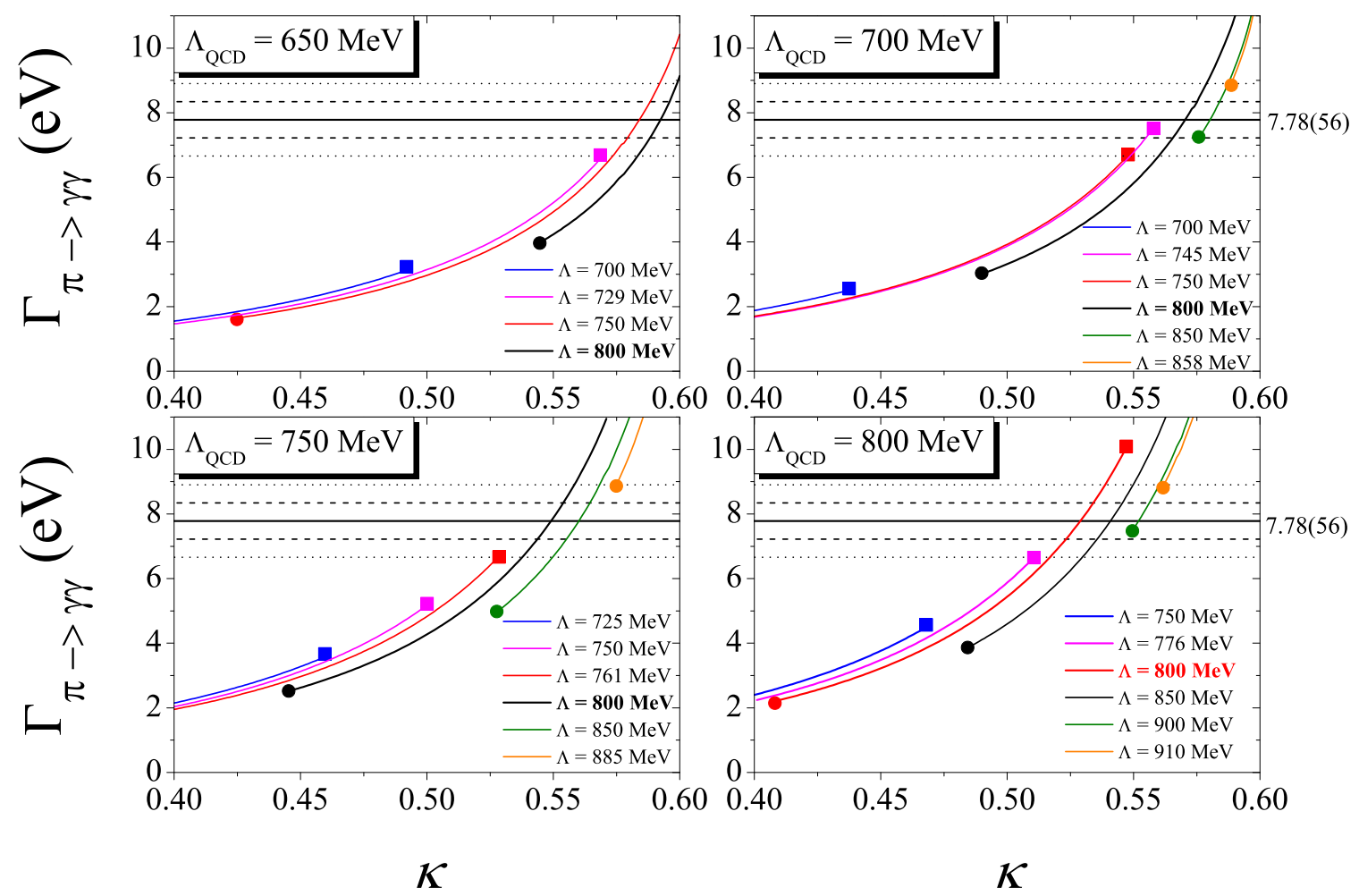

FIG. 3. $\Gamma_{\pi \rightarrow \gamma \gamma}$ as a function of $\kappa$ for several values of $\Lambda$ and $\Lambda_{Q C D}$. The circles and squares mean $M_{q}=300 \mathrm{MeV}$ and $M_{q}=400 \mathrm{MeV}$ respectively.

$\Lambda$ around $800 \mathrm{MeV}$ seems natural. If one wants to consider higher cut-off values, one should also include the vector and axial-vector currents in the model.

The decoupling type of propagator can be investigated setting $\kappa=0.5$ in Eq. (3). Then the gluon propagator becomes

$$
D\left(p^{2}\right)=\frac{Z}{p^{2}+M_{\text {gluon }}^{2}} ;
$$

$M_{\text {gluon }}$ takes the role of $\Lambda_{Q C D}$ and can be interpreted as an effective gluon mass. Following the same procedure as before, it follows that the model for $\kappa=0.5$ also reproduces $M_{\pi}, f_{\pi}$ and $\Gamma_{\pi \rightarrow \gamma \gamma}$, provided the gluon mass is adjusted as a function of the cut-off $\Lambda$. Our results are summarized in Table II and Fig. 4, for various cut-off values.

The effective gluon mass $M_{\text {gluon }}=\Lambda_{Q C D}$ is within typical values found in the literature, see [36] and references there in, but shows a strong dependence with the cut-off. $M_{\text {gluon }}$ is a linear function of $\Lambda$ - see Fig. 4. In what concerns the quark condensate, the model 


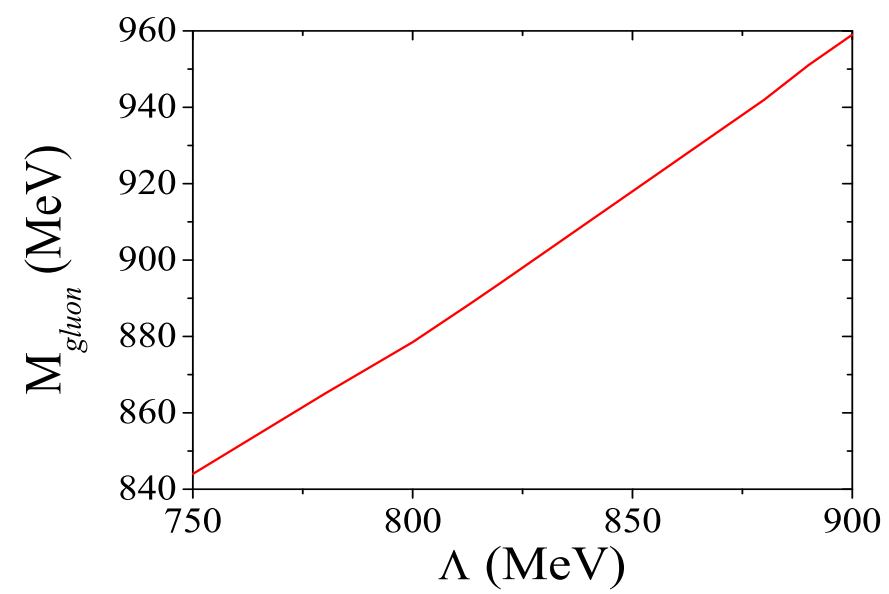

FIG. 4. $M_{\text {gluon }}(\Lambda)$ required to reproduce the experimental $\Gamma_{\pi \rightarrow \gamma \gamma}$.

shows that $\langle\bar{q} q\rangle$ increases with $M_{\text {gluon }}$, i.e with the cut-off $\Lambda$. For $\kappa=0.5$, to reproduce the experimental value of the condensate, i.e. to have $-\langle\bar{q} q\rangle^{1 / 3}=270 \mathrm{MeV}$, it turns out that the gluon mass is $M_{\text {gluon }}=878 \mathrm{MeV}$ for $\Lambda=800 \mathrm{MeV}$.

Finally, we would like to refer that the GMOR relation is satisfied always, i.e. for $\kappa>0.5$ and $\kappa=0.5$, within the same level of precision.

In this paper, the connection between QCD and the NJL model is explored to investigate possible links between the quenched gluon propagator and low energy hadronic phenomenology. The relation between the nonlocal model and QCD used is valid at the leading order level. It has been shown that nonlocal covariant extensions of the NJL model have the advantage, over the local theory, of the next-to-leading order corrections are relatively small [37]. Therefore, we expect that, at least qualitatively, our results will still be valid beyond the leading order.

Our main goal is to connect $D\left(p^{2}\right)$ with pion physics. The results discussed previously suggest that low energy physics is essentially blind to the gluon propagator at very low momenta. Indeed, the results show that one is free to use either the decoupling of scaling scenarios for $D\left(p^{2}\right)$, provided that the model parameters are chosen appropriately.

For a scaling-type scenario, $M_{\pi}$ and $f_{\pi}$ are not sensitive to the deep infrared gluon propagator. Indeed both values can be reproduced for $\kappa$ above and below 0.5. However, $\Gamma_{\pi \rightarrow \gamma \gamma}$ is sensitive to $\kappa$. Curiously, for $\Lambda=\Lambda_{Q C D}=800 \mathrm{MeV}$, the value of $\kappa$ which reproduces the experimental decay width, $\kappa=0.529$, is in perfect agreement with the infrared exponent 


\begin{tabular}{|c|c|c|c|c|c|}
\hline \hline$\Lambda[\mathrm{MeV}]$ & $M_{\text {gluon }}[\mathrm{MeV}]$ & $m_{q}[\mathrm{MeV}]$ & $M_{q}[\mathrm{MeV}]$ & $-\langle\bar{q} q\rangle^{1 / 3}[\mathrm{MeV}]$ & $G \Lambda^{2}$ \\
\hline \hline 750 & 843.8 & 4.5 & 460.9 & 264.2 & 6.03 \\
\hline 800 & 878.7 & 4.2 & 409.8 & 271.6 & 5.39 \\
\hline 813 & 888.5 & 4.1 & 400.0 & 273.5 & 5.27 \\
\hline 850 & 917.7 & 3.9 & 373.3 & 278.9 & 4.96 \\
\hline 900 & 959.1 & 3.6 & 345.7 & 286.3 & 4.66 \\
\hline
\end{tabular}

TABLE II. Summary of the results for $\kappa=0.5$.

measured from lattice QCD in [19, 24] and the estimate from time-independent stochastic quantization [38]. Of course, given the level of approximations used here, the good agreement is, probably, an happy coincidence.

For a decoupling-type scenario, the "measured" effective gluon mass is within typical values. From the values of the quark condensate, the massive gluon model seems to prefer a $M_{\text {gluon }} \sim 878 \mathrm{MeV}$.

The parameterizations discussed in this work gather the essential ingredients of the scaling and decoupling solutions of the DSE and the lattice propagators for $p \in[50,800] \mathrm{MeV}$. Besides the link with the infrared gluon propagator, the models analyzed also provide an effective nonlocal chiral quarks model and, in this sense, are a good starting point towards the construction of a more realistic nonlocal low-energy effective theory for QCD.

\section{ACKNOWLEDGMENTS}

The authors acknowledge financial support from F.C.T. under project CERN/FP/83644/2008 and (P.J.S.) grant SFRH/BPD/40998/2007. O.O acknowledges financial support from FAPESP.

[1] L. P. Faddeev, V. N. Popov, Phys. Lett B25, 29 (1967).

[2] V. N. Gribov, Nucl. Phys. B139, 1 (1978).

[3] See P. J. Silva, O. Oliveira, Nucl. Phys. B690, 177 (2004) and references therein.

[4] I. M. Singer, Comm. Math. Phys. 60, 7 (1978). 
[5] D. Zwanziger, Nucl. Phys. B323, 513 (1989).

[6] D. Zwanziger, Nucl. Phys. B345, 461 (1990).

[7] D. Dudal, et al., Phys. Rev. D78, 065047 (2008).

[8] D. Zwanziger, Nucl. Phys. B364, 127 (1991).

[9] D. Zwanziger, Nucl. Phys. B412, 657 (1994).

[10] D. Dudal, O. Oliveira, N. Vandersickel, Phys. Rev. D81 (2010) 074505

[11] C. Lerche, L. von Smekal, Phys. Rev. D65 (2002) 125006; J. M. Pawlowski, et al., Phys. Rev. Lett. 93, 152002 (2004); C. S. Fischer, M. R. Pennington, Phys. Rev. D73, 034029 (2006).

[12] C. S. Fischer J. Phys. G32 (2006) R253; R. Alkofer, L. von Smekal, Phys. Rep. 353 (2001) 281.

[13] A. C. Aguilar, A. A. Natale, P. S. Rodrigues da Silva, Phys. Rev. Lett. 90, 152001 (2003); A. C. Aguilar, J. Papavassiliou, Phys. Rev. D77, 125022 (2008); A. C. Aguilar, D. Binosi, J. Papavassiliou, Phys. Rev. D78, 025010 (2008); D. Binosi, J. Papavassiliou, JHEP 811, 63 (2008).

[14] D. Binosi, J. Papavassiliou, Phys. Rep. 479, 1 (2009).

[15] Ph. Boucaud, et al., arXiv:hep-ph/0507104; Ph. Boucaud, et al., Eur. Phys. J. A 31 (2007) 750; Ph. Boucaud, et al., JHEP 76 (2007) 0703; Ph. Boucaud, et al., JHEP 99 (2008) 0806; J. Rodríguez-Quintero arXiv:1005.4598

[16] D.B. Leinweber, et al., Phys. Rev. D58, 031501 (1998); E.-M. Ilgenfritz, et al., Braz. J. Phys. 37, 193 (2007); A. Cucchieri, T. Mendes, O. Oliveira, P. J. Silva, Phys. Rev. D76, 114507 (2007); I. L. Bogolubsky, et al., Phys. Rev. D77, 014504 (2008); O. Oliveira, P. J. Silva, E.-M. Ilgenfritz, A. Sternbeck, PoS (LATTICE2007) 323; I. L. Bogolubsky, et al., PoS (LATTICE2007) 290; A. Maas, Phys. Rev. D79, 014505 (2009).

[17] O. Oliveira, P. J. Silva, Braz. J. Phys. 37, 201 (2007).

[18] O. Oliveira, P. J. Silva, Pos (QCD-TNT09), 33 (2009).

[19] O. Oliveira, P. J. Silva, EPJ A31, 790 (2007).

[20] A. Cucchieri, T. Mendes, PoS (LATTICE2007) 297; O. Oliveira, P. J. Silva, PoS (LATTICE2005) 287; F. D. R. Bonnet, P. O. Bowman, D. B. Leinweber, A. G. Williams, J. M. Zanotti, Phys. Rev. D64, 034501 (2001).

[21] A. Cucchieri, T. Mendes, Phys. Rev. Lett. 100, 241601 (2008). 
[22] I. L. Bogolubsky, E.-M. Ilgenfritz, M. Müller-Preussker, A. Sternbeck, Phys.Lett. B676, 69 (2009).

[23] O. Oliveira, P. J. Silva, Phys. Rev. D79, 031501(R) (2009).

[24] O. Oliveira, P. J. Silva, Eur. Phys. J. C62, 525 (2009).

[25] Y. Nambu and G. Jona-Lasinio, Phys. Rev. 122, 345 (1961); Phys. Rev. 124, 246 (1961).

[26] H. Grigorian, Phys. Part. Nucl. Lett. 4, 223 (2007).

[27] M. Jamin, Phys. Lett. B 538 (2002) 71 arXiv:hep-ph/0201174.

[28] J. Bordes, C. A. Dominguez, P. Moodley, J. Penarrocha and K. Schilcher, JHEP 1005 (2010) 064 arXiv:1003.3358 [hep-ph]].

[29] C. Amsler et al. (Particle Data Group), Phys. Lett. B667, 1 (2008).

[30] S. M. Schmidt, D. Blaschke and Y. L. Kalinovsky, Phys. Rev. C50, 435 (1994).

[31] D. Blaschke, Yu.L. Kalinovski, P. Petrow, S.M. Schmidt, M. Jaminon, and B. Van den Bossche, Nucl. Phys. A592, 561 (1995).

[32] D. Blaschke, G. Burau, Y. L. Kalinovsky, P. Maris and P. C. Tandy, Int. J. Mod. Phys. A16, 2267 (2001).

[33] P. Costa, M. C. Ruivo and Yu. L. Kalinovsky, Phys. Lett. B560, 171 (2003); Phys. Rev. C70, $025204(2004)$.

[34] P. Costa, M. C. Ruivo and Yu. L. Kalinovsky, Phys. Lett. B577, 129 (2003); Phys. Lett. B581, 274 (E) (2004).

[35] J. Gronberg, et al., (CLEO Collaboration), Phys. Rev. D57, 33 (1998); C. Amsler et al. (Particle Data Group), Phys. Lett. B667, 1 (2008).

[36] O. Oliveira, P. Bicudo, arXiv:1002.4151,

[37] G. Ripka, Nucl. Phys. A683 (2001) 463; R.S. Plant and M.C. Birse, Nucl. Phys. A703 (2002) 717.

[38] D. Zwanziger, Phys. Rev. D67, 105001 (2003). 